\title{
Alteraciones histopatológicas de placenta en gestantes con preeclampsia y normotensas a 3660 msnm - Perú
}

Rossibel Juana Muñoz De la Torre rossibel.delatorre@unh.edu.pe DOI: https://orcid.org/0000-0003-1544-8863 Universidad Nacional de Huancavelica

Ena Carol Solano Huayra 2011321078@unh.edu.pe Universidad Nacional de Huancavelica

Jessica Ruiz Paitan 2012321058@unh.edu.pe Universidad Nacional de Huancavelica

Cesia Rebeca Zarate Cáceres cesiazaratec@gmail.com DOI: https://orcid.org/0000-0001-6168-5574 Universidad Nacional de Huancavelica

Edwin Jony Toral Santillán edwin.toral@unh.edu.pe https://orcid.org/0000-0001-7366-8472 Universidad Nacional de Huancavelica

Walter Ayvar Gutiérrez walteea50@yahoo.es Hospital Regional Zacarías Correa Valdivia de Huancavelica Huancavelica - Perú

\section{RESUMEN}

La preeclampsia es una enfermedad multisistémica del embarazo manifestada a partir de las 20 semanas (Pacheco Romero, 2002); el objetivo es determinar las alteraciones histopatológicas de la placenta en gestantes con pre eclampsia y normo tensas a 3660 msnm. Para ello se aplica un estudio observacional, prospectivo de corte transversal siguiendo un diseño comparativo donde se analizó 130 placentas distribuidos en dos grupos proporcionales aplicando la técnica de Bernischke y Driscol para que luego las láminas puedan ser leídos en un microscopio convencional y observar las alteraciones histopatológicas en cada grupo, ambos del Hospital Zacarías Correa Valdivia de Huancavelica, durante los meses de noviembre de 2019 a octubre de 2020. Los resultados muestran que las placentas de gestantes con preeclampsia a 3660 msnm a la macroscopía 
tienen en su mayoría inserción marginal con membranas fetales en el parénquima pletórico; a la microscopía con nódulos sinciciales el 90.8\%, infartos, microcalcificaciones, estromas congestivos y fibrosis marginal. En conclusión, las alteraciones histopatológicas halladas en la placenta con preeclampsia a $3660 \mathrm{msnm}$ demuestran un flujo uteroplacentario disminuidos, las mismas que deben tener prioridad en la atención materna perinatal en Huancavelica para evitar desenlaces fatales tanto para la madre como para el niño.

Palabras clave: alteraciones histopatológicas; placenta; preeclampsia 


\title{
Histopathological alterations of the placenta in pregnant women with preeclampsia and normotensive at 3660 altitude - Peru
}

\begin{abstract}
Preeclampsia is a multisystemic disease of pregnancy manifested after 20 weeks; The objective is to determine the histopathological alterations of the placenta in pregnant women with pre-eclampsia and normal tension at 3660 meters above sea level. For this, an observational, prospective cross-sectional study is applied following a comparative design where 130 placentas distributed in two proportional groups were analyzed applying the Bernischke and Driscol technique so that later the slides can be read in a conventional microscope and observe the histopathological alterations. in each group, both from the Zacarías Correa Valdivia Hospital in Huancavelica, during the months of November 2019 to October 2020. The results show that the placentas of pregnant women with preeclampsia at 3660 masl at macroscopy have mostly marginal insertion with fetal membranes in the full parenchyma; to microscopy with syncytial nodules $90.8 \%$, infarcts, microcalcifications, congestive stromas and marginal fibrosis. In conclusion, the histopathological alterations found in the placenta with preeclampsia at 3660 meters above sea level demonstrate a decreased uteroplacental flow, which should have priority in perinatal maternal care in Huancavelica to avoid fatal outcomes for both the mother and the child.
\end{abstract}

Keywords: histopathological alterations; placenta; preeclampsia

Artículo recibido: 15 abril 2021

Aceptado para publicación: 19 abril 2021

Correspondencia: rossibel.delatorre@unh.edu.pe

Conflictos de Interés: Ninguna que declarar 


\section{INTRODUCCIÓN}

La preeclampsia es una enfermedad hipertensiva propia del embarazo con compromiso multisistémico que ocurre después de las 20 semanas de gestación, caracterizada por vasoconstricción periférica y disminución de la distensibilidad arterial (Reyna-Villasmil, 2017); cuyo origen sigue siendo desconocido (Pacheco-Romero, 2017); sin embargo, parecen desencadenarse de la invasión anormal del trofoblasto de las arterias espirales de la decidua generando una remodelación defectuosa de lesiones arteriales (ReynaVillasmil, Briceño-Pérez, \& Santos-Bolívar, 2012) y por ende una hipoxia uteroplacentaria (Myatt, 2002) atenuando aún más la invasión del trofoblasto (Dekker \& Sibai, 2001).

La hipoperfusión uteroplacentaria genera subproductos de la peroxidación lipídica en el plasma sanguíneo posiblemente alterando la estructura y funcionabilidad de las membranas del endotelio y demás tejidos (Abad et al., 2012), a ello aunado la exposición a la altura mayores de 3000 msnm aumentan la probabilidad de daño en la perfusión uteroplacentaria (Gonzales, 2012), porque el desarrollo de la placenta tiene afinidad estrecha con la disponibilidad de oxígeno (Escudero \& Calle, 2006).

La vasculatura umbilicoplacentaria responde a las modificaciones del medio materno fetal, pudiendo ser inmediatas, mediadoras y duraderas con la finalidad de compensar las agresiones iniciales pero estos pueden cambiar a procesos disfuncionales de larga duración; es así que la hipoxia y el estrés oxidativo inducen a la disfunción endotelial reduciendo la perfusión placentaria(Herrera et al., 2014); estas respuestas placentarias hipóxicas alteran histopatológicamente a la placenta más aun en grandes altitudes, situación que motivó a desarrollar el presente estudio en gestantes con preeclampsia que residen a $3660 \mathrm{msnm}$.

\section{MATERIALES Y MÉTODOS}

La investigación corresponde a un estudio observacional, prospectivo, transversal aplicando un diseño descriptivo comparativo puesto que los datos provienen de fuentes primarias; se desarrolló con 130 placentas provenientes de dos grupos de parturientas con preeclampsia y normotensas atendidas en el Hospital Zacarías Correa Valdivia cuyas gestantes habitaron en los últimos seis meses en la cuidad de Huancavelica a 3660msnm, ambas distribuidas proporcionalmente y seleccionadas por tener embarazos únicos, a término y sin comorbilidad. 
La técnica empleada fue la observación permitiendo fijar a la placenta macro y microscópicamente, cuyo instrumento fue la ficha de observación estructurada acorde a la naturaleza del estudio validada cualitativamente por el juicio de expertos.

Las placentas o partes de ellas recolectadas fueron conservadas en formol al $10 \%$ hasta ser trasladados al laboratorio de la Universidad Nacional de Huancavelica, luego fueron lavadas y observadas macroscópicamente tomando mediciones con una regla y el peso con una balanza manual y observar la organización de cotiledones, la inserción del cordón umbilical y aspecto de las membranas para luego aplicando el protocolo de Bernischke y Driscol realizar cuatro cortes: placa corial, de la decidua, del rollo de la membrana coriónica y del cordón umbilical, con un grosor de 8 micras cada trozo, todas se fijan en formol al 10\%; después de 24 horas se procesó el tejido en el histoquinet, se incluyó en parafina realizando cortes de cinco micras del material seleccionado y finalmente se colocaron en láminas porta objetos para ser teñidos con la técnica de hematoxilina y eosina (Benirschke \& Driscoll, 1967).

Posterior a ello, las láminas fueron lecturadas a través del microscopio de luz convencionales del laboratorio clínico de la facultad de ciencias de la Salud, siendo validados los resultados por un experto en la materia.

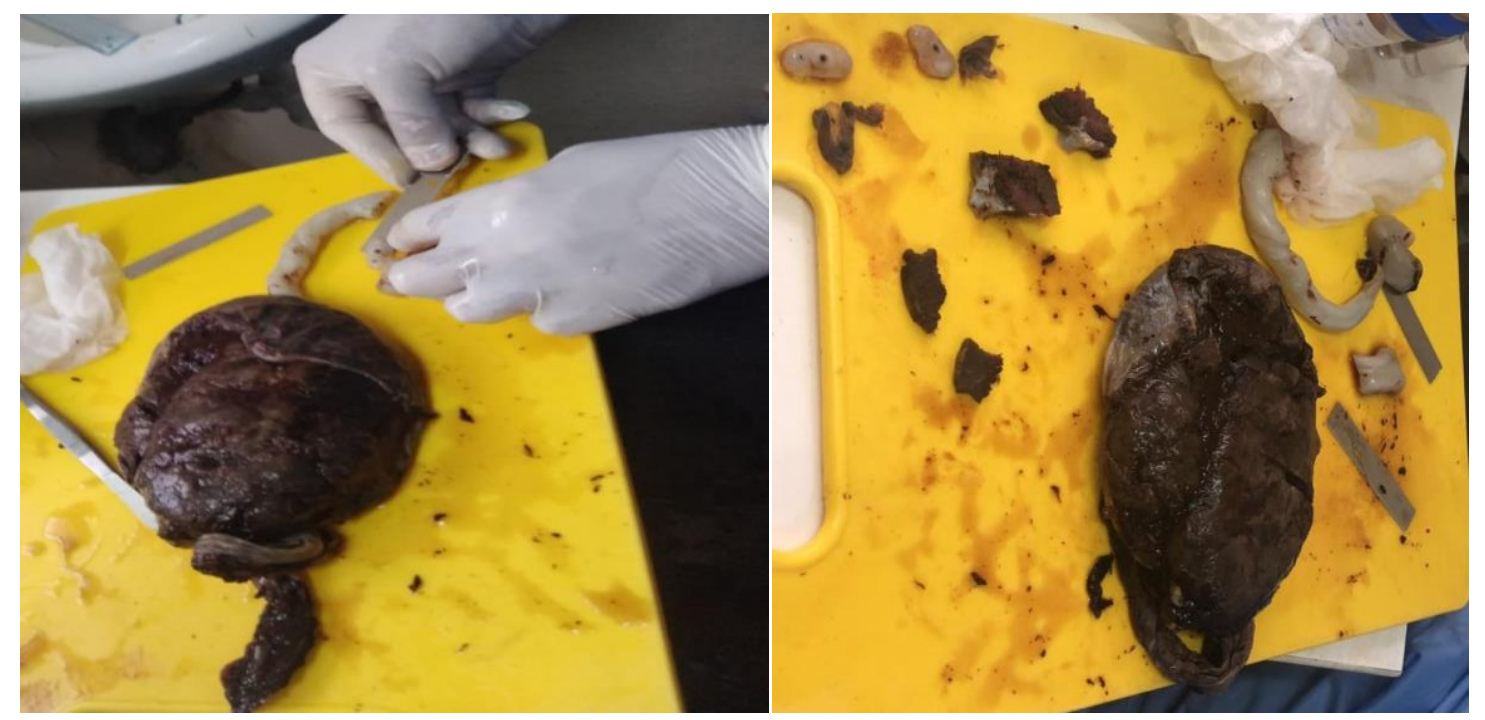

Gráfico A y B: (A) Identificación macroscópicas de la placenta, tomando medidas; (B) Cortes seriados de la placenta a nivel de la lámina corial, decidua, membrana coriónica y cordón umbilical. 


\section{RESULTADOS Y DISCUSIÓN}

Tabla N. 1: Características macroscópicas del disco placentario en gestantes con preeclampsia y normo tensas a $3660 \mathrm{msnm}$

\begin{tabular}{lcccccc}
\hline \multirow{2}{*}{$\begin{array}{c}\text { Disco } \\
\text { placentario }\end{array}$} & \multicolumn{2}{c}{ Gestante con Preeclampsia } & \multicolumn{2}{c}{ Gestante Normotensa } \\
\cline { 2 - 7 } & Media & Mediana & $\begin{array}{c}\text { Mínimo y } \\
\text { máximo }\end{array}$ & Media & Mediana & $\begin{array}{c}\text { Mínimo y } \\
\text { máximo }\end{array}$ \\
\hline Peso & $454.25 \mathrm{gr}$ & $452.7 \mathrm{gr}$ & $\begin{array}{c}300.21 \mathrm{gr} \\
460.2 \mathrm{gr}\end{array}$ & $498,90 \mathrm{gr}$ & $506,9 \mathrm{gr}$ & $457.3 \mathrm{gr}$ \\
Volumen & $458.3 \mathrm{~cm}^{3}$ & $436 \mathrm{~cm}^{3}$ & $320.7 \mathrm{~cm}^{3}$ & $495.6 \mathrm{~cm}^{3}$ & 498.2 & $305 \mathrm{~cm}^{3}$ \\
& & & $520.3 \mathrm{~cm}^{3}$ & & $\mathrm{~cm}^{3}$ & $720 \mathrm{~cm}^{3}$ \\
Espesor & $2.3 \mathrm{~cm}$ & $2.5 \mathrm{~cm}$ & $1.8 \mathrm{~cm}^{2}$ & $2.25 \mathrm{~cm}$ & $2.30 \mathrm{~cm}$ & $1.8 \mathrm{~cm}$ \\
& & & $3 \mathrm{~cm}$ & & & $2.6 \mathrm{~cm}$ \\
Diámetro & $15 \mathrm{~cm}$ & $14.8 \mathrm{~cm}$ & $13 \mathrm{~cm}$ & $14.9 \mathrm{~cm}$ & $18 \mathrm{~cm}$ & $15 \mathrm{~cm}$ \\
& & & $17 \mathrm{~cm}$ & & & $22 \mathrm{~cm}$ \\
\hline
\end{tabular}

Fuente: Ficha de observación aplicados a las placentas de gestantes con preeclampsia y normotensas a 3660 msnm.

El disco placentario y el volumen en gestantes con preeclampsia a 3660msnm es menor que en una normotensa y en cuanto al espesor y diámetro de las placentas, aparentemente sin diferencia alguna.

Tabla N.2: Características macroscópicas del cordón umbilical en gestantes con preeclampsia y normo tensas a $3660 \mathrm{msnm}$

\begin{tabular}{lcccc}
\hline \multirow{2}{*}{$\begin{array}{c}\text { Características del } \\
\text { cordón umbilical }\end{array}$} & \multicolumn{2}{c}{$\begin{array}{c}\text { Gestante con } \\
\text { preeclampsia }\end{array}$} & \multicolumn{2}{c}{ Gestantes normotensas } \\
\cline { 2 - 5 } & $\begin{array}{c}\mathbf{N}^{\circ} \\
(\mathbf{6 5 )}\end{array}$ & $\begin{array}{c}\mathbf{\%} \\
\mathbf{( 1 0 0 \% )}\end{array}$ & $\begin{array}{c}\mathbf{N}^{\circ} \\
\mathbf{( 6 5 )}\end{array}$ & $\begin{array}{c}\mathbf{\%} \\
\mathbf{( 1 0 0 \% )}\end{array}$ \\
\hline Inserción & 10 & $15.4 \%$ & 49 & $75.4 \%$ \\
Central & 0 & $0 \%$ & 4 & $6.2 \%$ \\
Excéntrico & 55 & $84.6 \%$ & 12 & $18.5 \%$ \\
Marginal & & & & \\
Color & 33 & $50.8 \%$ & 25 & $38.5 \%$ \\
Blanquecino & 30 & $46.2 \%$ & 38 & $58.5 \%$ \\
Opaco & 2 & $3.1 \%$ & 2 & $3.1 \%$ \\
Verdoso & & & & \\
\hline
\end{tabular}

Fuente: Ficha de observación aplicados a las placentas de gestantes con preeclampsia y normotensas a $3660 \mathrm{msnm}$.

El cordón umbilical de placentas en gestantes con preeclampsia en el 84.6\% tuvo inserción marginal y en el 58.5\% un aspecto opaco; en cambio en las gestantes normotensas en el $75.4 \%$ de inserción central y el $50.8 \%$ con un aspecto blanquecino. 
Tabla N. 3: Características macroscópicas de los cortes seriados de la placenta en gestantes con preeclampsia y normo tensas a $3660 \mathrm{msnm}$

\begin{tabular}{lcccc}
\hline \multirow{2}{*}{ Cortes seriados } & \multicolumn{2}{c}{$\begin{array}{c}\text { Gestante con } \\
\text { preeclampsia }\end{array}$} & \multicolumn{2}{c}{$\begin{array}{c}\text { Gestantes } \\
\text { normotensas }\end{array}$} \\
\cline { 2 - 5 } & $\mathbf{N}^{\circ}$ & $\%$ & $\mathbf{N}^{\circ}$ & $\%$ \\
\hline Parénquima & 7 & $10.8 \%$ & 59 & $90.8 \%$ \\
Rosado & 3 & $4.6 \%$ & 6 & $9.2 \%$ \\
Pálido & 55 & $84.6 \%$ & 0 & $0.0 \%$ \\
Pletórico & & & & \\
Lesiones & 28 & $43.1 \%$ & 16 & $24.6 \%$ \\
Calcificaciones & 12 & $18.5 \%$ & 5 & $7.7 \%$ \\
Infartos & 4 & $6.2 \%$ & 0 & $0 \%$ \\
Trombos de fibrina & 21 & $32.3 \%$ & 44 & $67.7 \%$ \\
Ninguno & & & & \\
\hline
\end{tabular}

Fuente: Ficha de observación aplicados a las placentas de gestantes con preeclampsia y normotensas a 3660 msnm.

Los cortes seriados de la placenta en gestantes con preeclampsia vistos macroscópicamente son: en el $84.6 \%$ parénquima pletórico con presencia de lesiones en el $43.1 \%$ de calcificaciones y $18.5 \%$ de infartos, en cambio en las normotensas el $90.8 \%$ el parénquima rosado con el $67.7 \%$ sin ninguna lesión.

Tabla N.4: Alteraciones microscópicas de los cortes seriados de la placenta en gestantes con preeclampsia y normo tensas a $3660 \mathrm{msnm}$

\begin{tabular}{|c|c|c|c|c|}
\hline \multirow[t]{2}{*}{ Hallazgos microscópicos } & \multicolumn{2}{|c|}{$\begin{array}{l}\text { Gestante con } \\
\text { preeclampsia }\end{array}$} & \multicolumn{2}{|c|}{$\begin{array}{c}\text { Gestantes } \\
\text { normotensas }\end{array}$} \\
\hline & $\mathbf{N}^{\circ}$ & $\%$ & $\mathbf{N}^{\circ}$ & $\%$ \\
\hline Fibrosis marginal & 64 & $98.5 \%$ & 1 & $1.5 \%$ \\
\hline Infartos significativos & 61 & $93.8 \%$ & 5 & $7.7 \%$ \\
\hline Nódulos sinciciales & 59 & $90.8 \%$ & 0 & $0.0 \%$ \\
\hline Estromas congestivos & 49 & $75.4 \%$ & 3 & $4.6 \%$ \\
\hline $\begin{array}{l}\text { Hiperplasia media intima de } \\
\text { los vasos }\end{array}$ & 18 & $27.7 \%$ & 10 & $15.4 \%$ \\
\hline Ninguno & 2 & $3.1 \%$ & 53 & $81.5 \%$ \\
\hline
\end{tabular}

Fuente: Ficha de observación aplicados a las placentas de gestantes con preeclampsia y normotensas a $3660 \mathrm{msnm}$.

Las alteraciones microscópicas de la placenta en gestantes con preeclampsia a 3660msnm en el $98.5 \%$ son fibrosis marginal, el $93.8 \%$ infartos, el $90.8 \%$ nódulos sinciciales y el $75.4 \%$ estromas congestivos; en cambio en las placentas de normotensas el $81.5 \%$ no se hallan alteraciones y solo $15.4 \%$ hiperplasia media íntima de los vasos. 
Los resultados del presente estudio albergaron a todas las gestantes con preeclampsia en su mayoría sin severidad tomando en cuenta la residencia a $3660 \mathrm{msnm}$, donde el disco placentario pesan por debajo de los 500 g.; con inserción marginal en el 84.6\%, parénquima pletórico y las siguientes alteraciones histopatológicas como fibrosis vellositaria marginal, infartos significativos, nódulos sinciciales y estromas congestivos probablemente como aspectos secundarios a isquemia placentaria no solo debido a la preeclampsia sino añadido la altitud en la que habitan; similares resultados fueron hallados por diversos estudios como Sodhi et al. al hallar el 65\% de infartos significativos no periféricos, hematoma retro placentario y calcificación en casos de preeclampsia grave (Sodhi, Mohan, Jaiswal, Mohan, \& Rathee, 1990); Soma, identificó infartos, con aumento de nódulos sincitiales, hipovascularidad de vellosidades, células del endotelio ampliadas y aterosis arterial en el lecho placentario (Soma, Yoshida, Mukaida, \& Tabuchi, 1982) y Gutiérrez halló placentas con aumento de células de hofbauer, cambios estromales y nodos sinciciales en más del 50\% (Gutiérrez et al., 2008), todos ellos apuntan al abundante material fibrinoide en las capas de Rohr y Nitabuch de la membrana basal (Aardema, Oosterhof, Timmer, Van Rooy, \& Aarnoudse, 2001) produciendo alteraciones endoteliales.

En casos de preeclampsia de inicio temprano, el volumen y el área de la superficie de las vellosidades terminales se vieron comprometidos provocando una reducción en el peso placentario, los cambios morfológicos de los componentes vasculares y vellosidades como características propias del inicio tardío de la preeclampsia (Egbor, Ansari, Morris, Green, \& Sibbons, 2006), sin embargo, tal afirmación es controversial con nuestros resultados, porque no se tomó en cuenta la altitud; puesto que la gestante con preeclampsia expuesta a grandes altitudes tiene mayor riesgo de resultados adversos (Gonzales, 2012); por otro lado, la hipovascularidad, fibrosis vellosa y proliferación severa del citotrofoblasto son resultado de la existencia del flujo uteroplacentario anormal precoz (Agalakova, Kolodkin, Adair, Trashkov, \& Bagrov, 2021) escaseando el aporte sanguíneo en la circulación cruzada donde la vellosidad está sumergida demostrando la disminución del flujo sanguíneo como causa significativa de los infartos, hipovascularidad (Brosens \& Renaer, 1972) (Castejón, 2000) además de las otras alteraciones observadas; finalmente la preeclampsia y la altura tienen un factor en común llamado estrés oxidativo (Burton \& Jauniaux, 2004) como prueba de placentación 
inadecuada provocando alteraciones histopatológicas en aparente estado de ausencia de signos de severidad.

\section{CONCLUSIÓN}

La placenta con preeclampsia mostró alteraciones histopatológicas siendo las más frecuentes fibrosis marginal, infartos, nódulos sinciciales y estromas digestivos a diferencia del grupo de placentas sin la enfermedad, ambos a una altura de 3600msnm.

\section{LISTA DE REFERENCIAS}

Aardema, M., Oosterhof, H., Timmer, A., Van Rooy, I., \& Aarnoudse, J. (2001). Uterine artery Doppler flow and uteroplacental vascular pathology in normal pregnancies and pregnancies complicated by pre-eclampsia and small for gestational age fetuses. Placenta, 22(5), 405-411.

Abad, C., Proverbio, T., Piñero, S., Botana, D., Chiarello, D. I., Marín, R., \& Proverbio, F. (2012). Preeclampsia, placenta, oxidative stress, and PMCA. Hypertension in Pregnancy, 31(4), 427-441.

Agalakova, N. I., Kolodkin, N. I., Adair, C. D., Trashkov, A. P., \& Bagrov, A. Y. (2021). Preeclampsia: Cardiotonic Steroids, Fibrosis, Fli1 and Hint to Carcinogenesis. International Journal of Molecular Sciences, 22(4), 1941.

Benirschke, K., \& Driscoll, S. G. (1967). The pathology of the human placenta Placenta (pp. 97-571): Springer.

Brosens, I., \& Renaer, M. (1972). On the pathogenesis of placental infarcts in preeclampsia. BJOG: An International Journal of Obstetrics \& Gynaecology, 79(9), 794-799.

Burton, G. J., \& Jauniaux, E. (2004). Placental oxidative stress: from miscarriage to preeclampsia. Journal of the Society for Gynecologic Investigation, 11(6), 342352.

Castejón, O. (2000). La microscopia electrónica de barrido del infarto placentario. Salus, 19-24.

Dekker, G., \& Sibai, B. (2001). Primary, secondary, and tertiary prevention of preeclampsia. The Lancet, 357(9251), 209-215.

Egbor, M., Ansari, T., Morris, N., Green, C. J., \& Sibbons, P. D. (2006). Morphometric placental villous and vascular abnormalities in early- and late-onset pre-eclampsia with and without fetal growth restriction. BJOG : an international journal of 
obstetrics and gynaecology, 113(5), 580-589. doi:10.1111/j.14710528.2006.00882.x

Escudero, C., \& Calle, A. (2006). [Iron, oxygen and placental development in the etiology of preeclampsia. Effects of high altitude in Ecuador]. Revista médica de Chile, 134(4), 491-498. doi:10.4067/s0034-98872006000400014

Gonzales, G. F. (2012). [Impact of high altitude on pregnancy and newborn parameters]. Revista Peruana de Medicina Experimental y Salud Publica, 29(2), 242-249. doi:10.1590/s1726-46342012000200013

Gutiérrez, G. R., Maldonado, H. A. V., Sashida, P. M., López, A. H., Salim, P. C., \& de León, A. L. P. P. (2008). Alteraciones histopatológicas placentarias en la hipertensión gestacional. Ginecologia y Obstetricia de Mexico, 76(11).

Herrera, E. A., Krause, B., Ebensperger, G., Reyes, R. V., Casanello, P., Parra-Cordero, M., \& Llanos, A. J. (2014). The placental pursuit for an adequate oxidant balance between the mother and the fetus. Front Pharmacol, 5, 149. doi:10.3389/fphar.2014.00149

Myatt, L. (2002). Role of placenta in preeclampsia. Endocrine, 19(1), 103-111.

Pacheco-Romero, J. (2017). Introducción al simposio sobre preeclampsia. Revista Peruana de Ginecología y Obstetricia, 63(2), 199-206.

Pacheco Romero, J. (2002). Ginecología y Obstetricia. Mad Corp. SA Perú.

Reyna-Villasmil, E. (2017). Factores anti-angiogénicos y preeclampsia. Revista de Obstetricia y Ginecología de Venezuela, 77(4).

Reyna-Villasmil, E., Briceño-Pérez, C., \& Santos-Bolívar, J. (2012). Alteración de la placentación profunda en la preeclampsia. Revista de Obstetricia y Ginecología de Venezuela, 72(4), 269-276.

Sodhi, S., Mohan, H., Jaiswal, T., Mohan, P., \& Rathee, S. (1990). Placental pathology in pre-eclampsia eclampsia syndrome. Indian journal of pathology \& microbiology, 33(1), 11-16.

Soma, H., Yoshida, K., Mukaida, T., \& Tabuchi, Y. (1982). Morphologic changes in the hypertensive placenta. Contributions to gynecology and obstetrics, 9, 58-75. 